\title{
Interleukin-13, Interleukin-10, Interferon- $\gamma$ and IDO Production in Response to Home Dust Mites in Allergic Asthma
}

\author{
Cityta Putri Kwarta ${ }^{1}$, Heri Wibowo ${ }^{2,}$, Yordan Khaedir $^{3}$ Iris Rengganis $^{4}$, Hanny Siti Nuraeni ${ }^{1}$ \\ ${ }^{1}$ Magister Program in Biomedical Sciences, Universitas Indonesia, Jl. Salemba Raya No.5, Jakarta, Indonesia \\ ${ }^{2}$ Department of Parasitology, Faculty of Medicine, Universitas Indonesia, J1. Salemba Raya No.5, Jakarta, Indonesia \\ ${ }^{3}$ Faculty of Medicine, Universitas Indonesia, Jl. Salemba Raya No.5, Jakarta, Indonesia \\ ${ }^{4}$ Division of Allergy Immunology, Department of Internal Medicine, Universitas Indonesia, Dr. Cipto Mangunkusumo Central General Hospital, \\ Jl. Diponogoro No.71, Jakarta, Indonesia \\ *Corresponding author. E-mail: bowoheri04@gmail.com
}

Received date: Jan 12, 2019; Revised date: Mar 11, 2019; Accepted date: Mar 13, 2019

\section{Abstract}

$\mathrm{B}$ ACKGROUND: Allergic asthma is a degenerative atopic disease caused by allergic or hypersensitivity type-1. More than $50 \%$ of people with allergic asthma are caused by the presence of house dust mites (HDMs) allergens.

METHODS: The cellular immunity response was evaluated through a peripheral blood mononuclear cell (PBMC) culture isolated from blood, using the ficoll gradient technique. Subjects were atopic asthma groups and non-atopic asthma groups. PBMC from each subject cultured was stimulated with $\mathrm{HDMs}$ allergen, then incubated in a $\mathrm{CO}_{2} 5 \%$ incubator, $37^{\circ} \mathrm{C}$ for 72 hours. With the multiplex assay method, interferon (IFN)- $\gamma$, interleukin (IL)-13 and IL-10 were measured, meanwhile indoleamine 2,3-dioxygenase level (IDO) was measured by the enzyme-linked immunosorbent assay (ELISA) sandwich methods.

RESULTS: The IFN- $\gamma$ production in the supernatant of PBMC cultures was stimulated by phytohemagglutinin (PHA), Roswell Park Memorial Institute (RPMI) medium and allergens. The IFN- $\gamma$ production in allergen-stimulated supernatants showed higher level of IFN- $\gamma$ in the non-

\section{Introduction}

Allergic diseases are the most common disorders of immunity in the entire world. More than $30 \%$ of the world population has allergic symptoms whose pattern of abnormalities atopic group $(4,681,455 \pm 3,434,851)$ than atopic group $(4,363,300 \pm 2,067,941)$ even though it was not statistically significant $(p=0.078)$. There were no differences between the mean of IL-13 production in atopic asthma group and non-atopic group. The IL-10 production in allergenstimulated supernatants was shown to be higher in nonatopic group and were statistically significantly different $(p=0.015)$. The IDO production in allergen-stimulated supernatants was shown to be higher in the non-atopic group $(272,231 \pm 269,564)$ than in the actopic group $(13,273 \pm 400)$, and it was significantly different $(p=0.007)$.

CONCLUSION: Cellular immune profile of subjects with allergic asthma to Dermatophagoides pterronyssinus (Der p) is characterized by a type- 2 inflammatory response that is dominant compared to type- 1 inflammation (higher IL-13 ratio compared to IFN- $\gamma$ ) and to the role of anti-inflammation (higher IL-13 ratio compared to IL-10). The decline in IDO production in allergic asthma subjects to Der $\mathrm{p}$ is thought to be related to the low cellular immune response in expressing IFN- $\gamma$ compared to IL-13.

KEYWORDS: interleukin-13, interleukin-10, IDO, PBMC, asthma

Indones Biomed J. 2019; 11(2): 194-9 is categorized as type-1 hypersensitivity.(1) In the last decade, diseases that are based on allergic mechanisms such as rhinitis, bronchial asthma, and prevalence dermatitis continue to increase. The prevalence of asthma in the world has increased in the past few decades, estimated to increase to 400 million by 2025.(2,3) Approximately $50-80 \%$ of 
the incidence of atopic asthma in the world is triggered due to a hypersensitive response to allergens from house dust mite (HDMs). Meanwhile, 90\% of asthma patients in Indonesia are vulnerable to exposure to house dust and HDMs. HDMs globally exist in almost all regions and are a significant factor underlying bronchial asthma allergies, making them the largest source of allergens in the room. HDMs affect $2 \%$ from the world population, the genus that has the greatest influence in Indonesia with high humidity is Dermatophagoides pteronyssinus (Der p) of $85 \%$ and Dermatophagoides farinae (Der f) of $47 \% .(2,3)$

The pattern of the balance of $\mathrm{T}$ Helperl (proinflammatory type-1), T Helper 2 (pro-type 2 inflammation) and the control function of the regulator $\mathrm{T}$ expressing antiinflammation are referenced as factors that are closely related to pathogenesis. The difference in immunity sensitization of asthma and non-atopic atopies (normal) to allergic symptoms after exposure to HDMs were related to a reduced regulatory function of regulatory $\mathrm{T}$ cells that work through anti-inflammatory pathways. Atopy has an allergenspecific memory Th cell contrast pattern.(3) HDMs allergy Th2 cells play an important role in allergic inflammatory responses including production of immunoglobulin $\mathrm{E}$ (IgE), recruitment of eosinophils in tissues, mucus production, ease of endothelium to recruit inflammatory cells into infected lungs, and modulate contractions of respiratory smooth muscles. One of the regulatory roles of regulatory $\mathrm{T}$ cells mediated by interactions with dendritic cells, this interaction will produce indoleamine 2,3-dioxygenase (IDO) molecules which act as enzymes to control lymphocyte cell growth. (3) It is expected that the results of this study can contribute several approaches in the development of management of cases of bronchial asthma due to allergies.

\section{Methods}

The population of this study was moderate persistent bronchial asthma and non-atopic asthma subjects. The inclusion criteria were subjects aged 30-59 years, living in Purwokerto for at least 3 years, and had a history of moderate persistent bronchial asthma (for asthma atopic subjects), or had no history of asthma and atopy (for nonatopic subjects). The feasibility of the research ethics study had been approved from the Research Ethics Committee of the Faculty of Medicine, Universitas Indonesia, No. 1251/ UN2.F1/ETIK/2018.

The subjects were tested for skin prick test (SPT) with HDMs Der $p$ allergen (Stallergenes Company, Kamp-
Lintfort, France). This test was performed on the volar area of the forearm with a distance of at least 2 $\mathrm{cm}$ from the fold of the elbow and wrist. The superficial layer of the skin was pierced using a special needle. The reaction was considered positive if itching and erythema were confirmed by the presence of a distinctive induration that could be seen and touched. The largest diameter (D) and the smallest diameter (d) is measured by the reaction expressed as size $(D+d) / 2$. Measurements were made by circling induration with a pen and pasted on a paper before the diameter measured. $(4,5)$

Each subject was taken venous blood to isolate mononuclear cells. PBMCs were blood cells that have nuclei, such as lymphocytes, monocytes and macrophages. The principle of PBMC isolation were following method from Fiqoll Histopaque (Sigma Aldrich, St. Louis, USA), where the difference or density gradient between the cell and the medium were differentiate into several layers through a centrifugation process.(6) PBMCs were given 3 stimulants, namely Phytohemagglutinin (PHA) (Sigma Aldrich) as a positive control, Roswell Park Memorial Institute (RPMI) medium (Sigma Aldrich) as a negative control, and allergen extract Der p (Stallergenes Company). About 500.000 cells/ $\mathrm{mL}$ PBMCs were cultured on microwell plate 96 wells and incubated in $5 \% \mathrm{CO}_{2}$ incubator, $37^{\circ} \mathrm{C}$ for 72 hours. After 72 hours, the supernatant was taken from each PBMC culture by centrifugation.

Measurement of interleukin (IL)-13, IL-10 and interferon (IFN)- $\gamma$ cytokines in the supernatant were done by using Multiplex Immunoassay Luminex (Thermo Fisher Scientific, Waltham, USA) with the results of the data in the form of net median fluorescent intensity (MFI) values then converted through a regression curve from each cytokine to obtain cytokines in units of $\mathrm{pg} / \mathrm{mL}$. The measurement of IDO levels in the supernatant were done by using the sandwich enzyme-linked immunosorbent assay (ELISA) (Wuhan USCN Business Co, Houston, USA) method with the results of the data in the form of optical density (OD) and then converted through a standard curve from each sample to obtain cytokines in $\mathrm{ng} / \mathrm{mL}$ unit.

Data analysis was done by using Windows 8 Statistical Package for Social Sciences (SPSS) software version 22 (IBM, New York, USA) to analyze the relationship of variables between patterns of activation of IL-13, IL-10, IFN- $\gamma$ and IDO for people with asthma due to HDMs and non-asthma sufferers because of HDMs. To compare the cellular responses to HDMs allergens from asthma atopic and non-atopic subjects as controls, Mann-Whitney nonparametric test was done. Meanwhile, non-parametric 
Spearman Rho correlation test was done to determine the magnitude of allergic events to cellular responses.

\section{Results}

This in vitro study was conducted on the PBMC culture. Total number of subjects in this study was 46 subjects, which consist of 25 moderate persistent asthma and positive SPT against allergens (atopic asthma) subjects and 21 subjects who did not suffer from negative asthma and SPT on allergens. The characteristics of the subject of this study was shown in Table 1.

The results of IFN- $\gamma$ levels measurement from PBMC cultures stimulated by PHA (as positive control), RPMI (as negative control), and allergen Der $\mathrm{p}$ (as allergen factors) in the atopic and non-atopic groups were shown in Figure 1 . The mean IFN- $\gamma$ production value in the positive control was not significantly different between groups $(p=0.903)$. In the negative control group, IFN- $\gamma$ production was higher in the non-atopic group $(p=0.08)$. Meanwhile and in the allergen-stimulated supernatants group, the production of IFN- $\gamma$ was found to be higher in the non-atopic group even though it was not statistically significant $(p=0.078)$. The comparison of stimulant administration PHA, RPMI and Der $\mathrm{p}$ in the atopic and non-atopic asthma groups showed very significant differences (Kruskal Wallis Test, $p=0.000$ ).

The measurement result of IL-13 levels from PBMC cultures stimulated by PHA, RPMI and Der $p$ allergens in the
Table 1. Characteristics of the research subjects.

\begin{tabular}{lcc}
\hline \multicolumn{1}{c}{ Characteristic } & Total (n) & Percentage (\%) \\
\hline Gender & & \\
$\quad$ Male & 14 & 30.4 \\
$\quad$ Female & 32 & 69.6 \\
Age, average & 45 & \\
SPT & & \\
$\quad$ Positive & 25 & 54.3 \\
$\quad$ Negative & 21 & 45.7 \\
Clinical Sign & & \\
$\quad$ Asthma & 25 & 54.3 \\
$\quad$ Non-asthma & 21 & 45.7 \\
Group & & \\
$\quad$ Atopic asthma & 25 & 54.3 \\
$\quad$ Non-atopic asthma & 21 & 45.7 \\
\hline
\end{tabular}

atopic and non-atopic asthma groups were shown in Figure 2. Figure 2 showed that the mean of IL-13 production in positive controls ( $p=0.522$ ), negative controls $(p=0.475$ ) and allergens ( $p=0.523$ ) were not significantly different between the atopic and non-atopic asthma. But when looking at the mean values IL-13 production, the atopic asthma group produced more IL-13 compared to the nonatopic group.

The measurement results of IL-10 levels from PBMC cultures stimulated by PHA, RPMI and Der $\mathrm{p}$ allergens in the atopic and non-atopic asthma groups were shown in Figure 3. Allergen-stimulated supernatants Der $p$ showed IL-10 production results were higher in the non-atopic group and were statistically significantly different $(p=0.015)$.

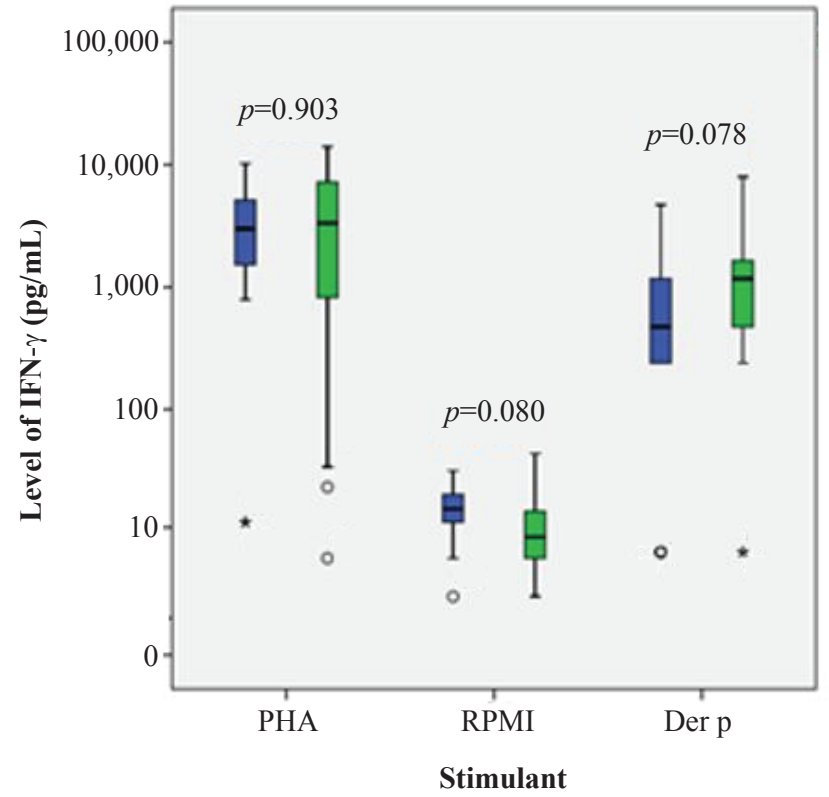

Atopic asthma

Non-atopic asthma
Figure 1. IFN- $\gamma$ levels from PBMC cultures stimulated by PHA (as positive control), RPMI (as negative control), and Der $p$ allergens (as allergens) in the atopic and nonatopic asthma group. 


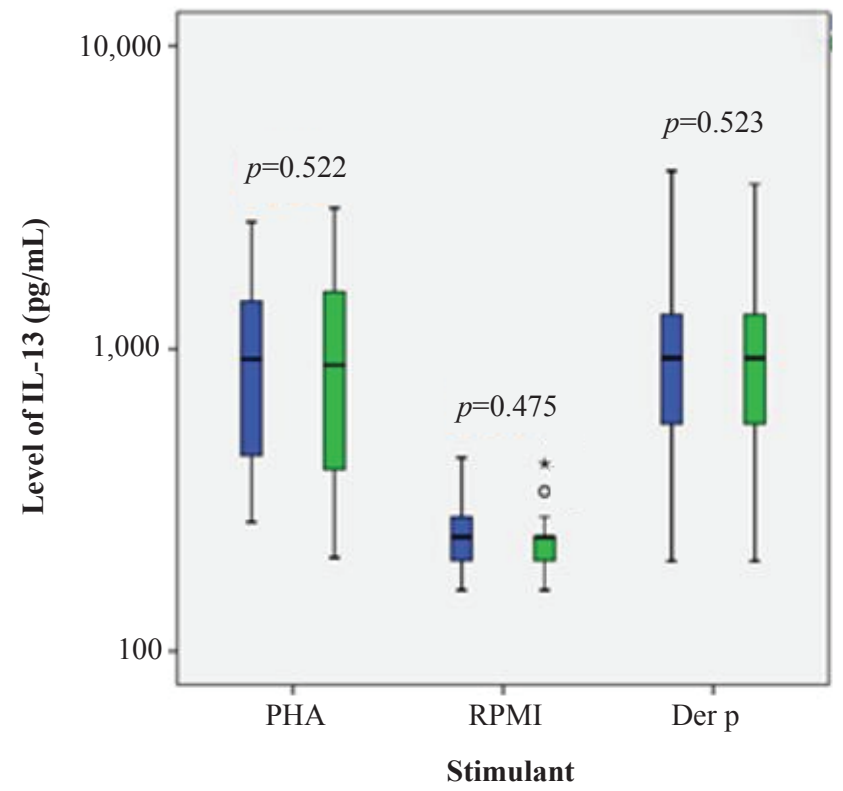

The measurement results of IDO levels from PBMC cultures stimulated by PHA, RPMI and Der $p$ allergens in the atopic and non-atopic asthma groups were shown in Figure 4. From Figure 4, it was known that the mean IDO production values in positive controls $(p=0.462)$ and negative controls $(p=0.549)$ did not differ significantly between groups of atopic and non-atopic asthma subjects. But Der $\mathrm{p}$ allergen stimulated supernatants showed higher IDO production in the non-atopic group and statistically significantly different $(p=0.007)$. The comparison of stimulant administration PHA, RPMI and Der $p$ in the atopic and non-atopic asthma groups showed mean differences that tended to be higher for stimulant Der $\mathrm{p}$, although statistically not significant (Kruskal Wallis Test, $p=0.084$ ).

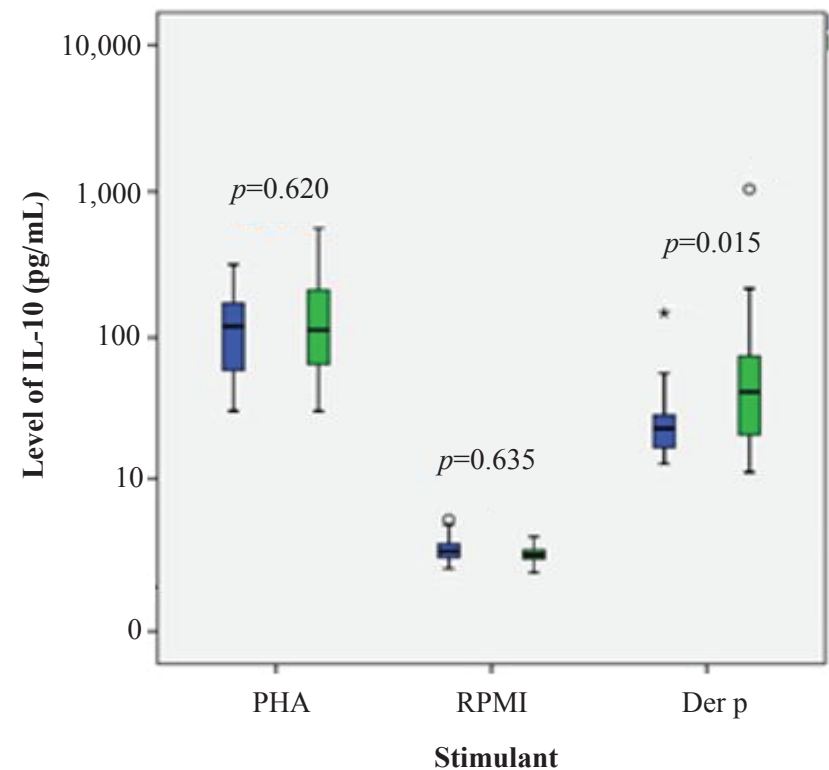

Atopic asthma

a Non-atopic asthma
Figure 2. IL-13 levels from PBMC cultures stimulated by PHA (as positive control), RPMI (as negative control), and Der $p$ allergens (as allergens) in the atopic and nonatopic asthma group.

\section{Discussion}

The measurement results of IFN- $\gamma$ levels from PBMC cultures stimulated by PHA, RPMI, Der $\mathrm{p}$ and allergens showed that IFN- $\gamma$ levels of positive control, negative controls, and allergens differing significantly in both the atopic and non-atopic asthma groups. This shows that PBMC has a good response to stimulation with PHA and Der $p$ allergens. The response to PHA as positive control is higher because PHA is a nonselective mitogen (can affect various types of lymphocyte subpopulations), while allergens are selective in $\mathrm{T}$ cell lymphocytes. $(7,8)$ Although the comparison of intergroup levels did not show a statistically

Atopic asthma

a Non-atopic asthma

Figure 3. IL-10 levels from PBMC cultures stimulated by PHA (as positive control), RPMI (as negative control), and Der $p$ allergens (as allergens) in the atopic and nonatopic asthma group. 


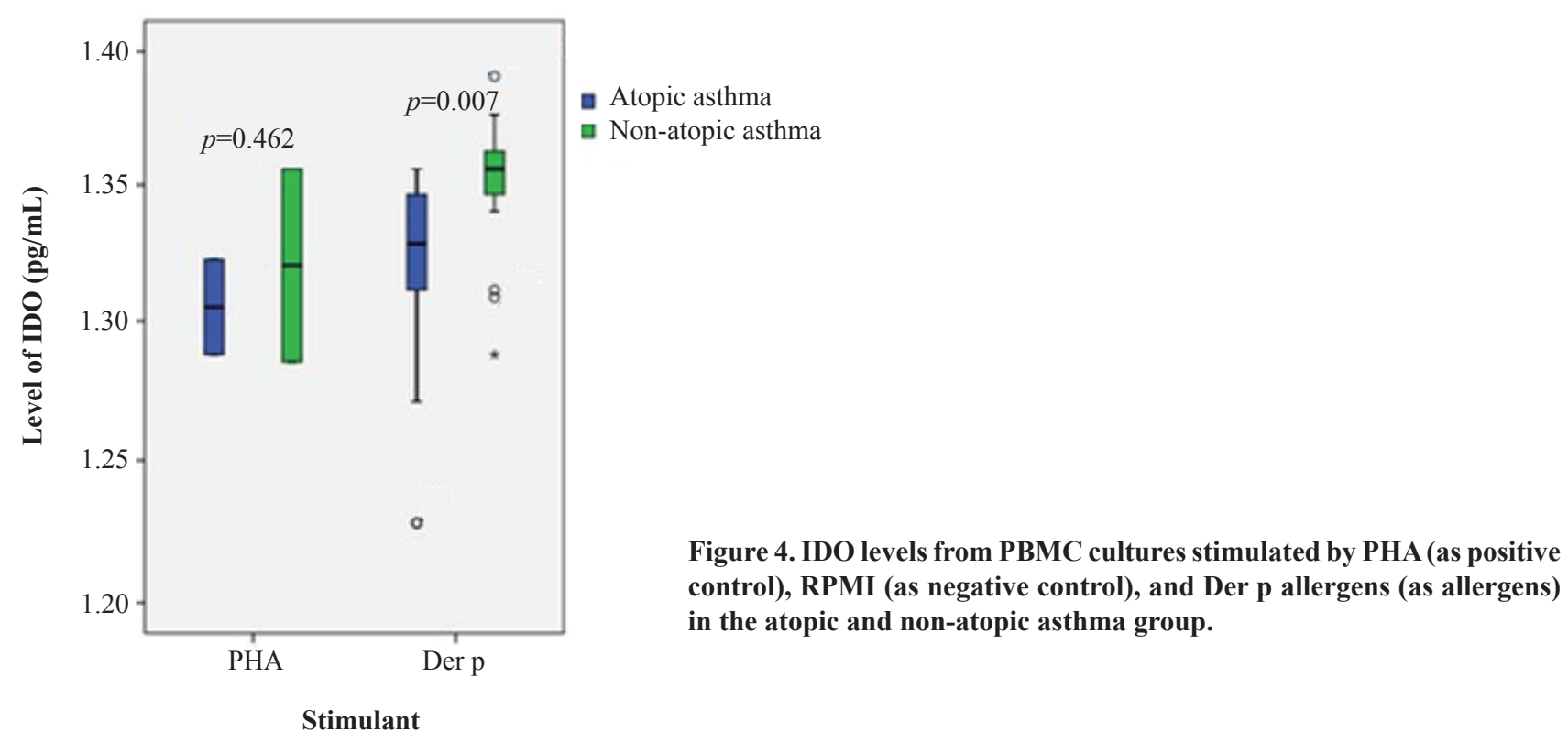

significant difference, the trend pattern for producing IFN- $\gamma$ cytokines was higher in the nonatopic group compared with the asthma atopy group.

Der $\mathrm{p}$ allergens in the form of cysteine proteases have been demonstrated in the previous study to reduce the tight junction in epithelial cells of healthy subjects and can activate protease-activated receptor (PAR)2 thereby inducing E-cadherin disruption in intercellular contacts. (9) This facilitates allergens to submucosal cells and increases allergic inflammation. The decrease in E-cadherin in the bronchial epithelium increases the expression of proinflammatory factors and promotes Th1 cell differentiation. In addition, activation of PAR2 by protease induces intracellular signaling activity including nuclear factor-KB (NFKB) and extracellular kinase (ERK) signaling pathways and there is an increase in IFN- $\gamma$ proinflammatory cytokines as the main effectors.(9-11)

In the atopic asthma group, IL-13 levels, which are Th2 proinflammatory cytokines, were seen to be higher than those of the nonatopic group even though the differences did not appear to be statistical, but it could be seen the tendency of different IL-13 activation patterns in the two groups. This is in line with a previous research. It was found that Der p can activate toll-like receptor (TLR)4 signal transduction so that it increases the expression of IL-6 and major histocompatibility complex (MHC) II which helps the process of differentiating naïve T cells into cells Th2.(10,12) This is confirmed by a study, which states that Der $\mathrm{p}$ which has cystein protease allergens can increase the polarization of naive T cells into Th2 cells and increase the production of proinflammatory cytokines Th2. $(9,13)$ The secretion of Th2 cytokines has the clinical effect of increasing eosinophil recruitment, mast cell activation, and $\mathrm{B}$ cell differentiation until class-switching becomes IgE. The levels of cytokine IL-13 are also a marker that the inflammatory reaction that occurs has entered the chronic phase. $(9,11)$ Non-significant conditions on IL-13 levels can be caused by the effects of asthma exercises carried out by research subjects for 4 months. Physical exercise can reduce Th2 proinflammatory cytokines and increase anti-inflammatory cytokines as reported by other studies that was done before. $(14,15)$

After analyzing IL-10 activation patterns in two groups of subjects, it was found that a higher increase in IL-10 levels in the non-atopic group was found compared to the atopyic asthma group. Increasing levels of antiinflammatory cytokines IL-10 can inhibit the production of proinflammatory cytokines through a direct mechanism that is towards the immune system effector targets and also an indirect mechanism that modulates the function of the immune system, for example preventing differentiation of dendritic cells. This will inhibit the co-stimulatory process, antigen presentation, and decrease the ability of chemokine secretion. In addition, increased production of antiinflammatory cytokines can also be caused by continued proinflammatory production.(16) This has been proved before that low IL-10 levels correlate strongly with the occurrence of the pathogenesis of asthma.(17)

The same thing was shown in IDO measurements from PBMC cultures stimulated by RPMI, PHA, and Der $p$ allergens that IDO levels in the non-atopic group increased significantly compared to the atopic group. PBMC cells cultured with stimulant antigens derived from Der $p$ 
allergens can respond in the form of IDO expression, but the results can be different if stimulated with other antigens because the response is the memory PBMC cell response with secondary exposure so that it is specific. $(8,11)$ is an enzyme and limits the level of tryptophan (Trp) along the kynurenine (Kyn) pathway. IDO is widely expressed in various types of cells, including leukocytes and APCs.(18)

IDO is induced in dendritic (DC) cells, which limits inflammation and prevents excessive host responses. Low IDO activity has previously been observed in asthma and atopic non-asthma patients.(19,20) In several studies, it was explained that IDO inhibits airway proinflammation mediated by Th2 cells, but does not seem to influence tolerance to inhibition in the immune response of proinflammatory cells Th1.(20,21,22)

\section{Conclusion}

Cellular immune profile of subjects with allergic asthma to Dermatophagoides pterronyssinus (Der p) is characterized by a type- 2 inflammatory response that is dominant compared to type-1 inflammation (higher IL-13 ratio compared to IFN- $\gamma$ ) and to the role of anti-inflammation (higher IL-13 ratio compared to IL-10). The decline in IDO production in allergic asthma subjects to Der $p$ is thought to be related to the low cellular immune response in expressing IFN- $\gamma$ compared to IL-13.

\section{References}

1. Reithofer M, Jahn-schmid. Allergens with protease activity from house dust mites. Int J Mol Sci 2017; 18: 1368. doi: 10.3390/ ijms18071368.

2. Curti A, Trabanelli S, Salvestrini V, Baccarani M, Lemoli RM. The role of indoleamine 2,3-dioxygenase in the induction of immune tolerance: focus on hematology. Blood. 2018; 113: 2394-402.

3. Wiest M, Upchurch K, Yin W, Ellis J, Xue Y, Lanier B, et al. Clinical implications of $\mathrm{CD} 4+\mathrm{T}$ cell subsets in adult atopic asthma patients. Allergy Asthma Clin Immunol. 2018; 14: 1-14. doi: 10.1186/ s13223-018-0231-3.

4. Svennberg K, Wadso L. House Dust Mites in Beds and Bedrooms. Sweden: Lund University; 2017

5. Ito T, Hirose K, Norimoto A, Tamachi T, Yokota M, Saku A, et al. Dectin-1 plays an important role in house dust mite-induced allergic airway inflammation through the activation of $\mathrm{CD} 11 \mathrm{~b}+$ dendritic cells. J Immunol. 2018; 198: 61-70.

6. Nazarpour R, Zabihi E, Alijanpour E, Abedian Z, Mehdizadeh H, Rahimi F. Optimization of human peripheral blood mononuclear cells (PBMCs) cryopreservation. Int J Mol Cell Med. 2012; 1: 8893.

7. Owen JA, Punt J, Strandford SA, Jones PP. Kuby Immunology. 7th Edition. New York: W. H. Freeman and Company; 2013.

8. Tsai C, Mukai K, Robinson PV, Gray MA, Waschmann MB, Lyu SC, et al. Isotype-specific agglutination-PCR (ISAP): a sensitive and multiplex method for measuring allergen-specific IgE. J Allergy Clin Immunol. 2018; 141: 1901-4.e15.

9. Parulekar AD, Kao CC, Diamant Z, Hanania NA. Targeting the interleukin-4 and interleukin-13 pathways in severe asthma: current knowledge and future needs. Clin Transl Allergy. 2018; 24: $50-5$.

10. Huang FL, Liao EC, Yu SJ. House dust mite allergy: Its innate immune response and immunotherapy. Immunobiology. 2018; 223: 300-2.

11. Campana R, Dzoro S, Mittermann I, Fedenko E, Elisyutina O, Khaitov $\mathrm{M}$, et al. Molecular aspects of allergens in atopic dermatitis. Curr Opin Allergy Clin Immunol. 2017; 17: 269-77.

12. Kenneth M. Janeway's Immubiology. 8th Edition. New York: Garland Science; 2016.

13. Delves PJ. MSD Manual Profession Version [Internet]. Overview of Allergic and Atopic Disorders [cited 2018 Dec 1]. Available from: https:/www.msdmanuals.com/professional/immunology-allergicdisorders/allergic,-autoimmune,-and-other-hypersensitivitydisorders/overview-of-allergic-and-atopic-disorders.

14. Dorneles GP, Haddad DO, Fagundes VO, Vargas BK, Kloecker A, Romão PRT, et al. High intensity interval exercise decreases IL-8 and enhances the immunomodulatory cytokine interleukin-10 in lean and overweight-obese individuals. Cytokine. 2016; 77: 1-9. doi: 10.1016/j.cyto.2015.10.003.

15. Suzuki K. Cytokine response to exercise and its modulation. Antioxidants. 2018; 7: 17. doi: 10.3390/antiox7010017.

16. Patricia H. Exploring the Effect of Sublingual Immunotheraphy on Lung Tissue-Resident Memory $\mathrm{T}$ Cells in Treatment of Allergic Asthma. Williamstown: Williams College; 2016.

17. Calderón MA, Linneberg A, Kleine-Tebbe J, De Blay F, Hernandez Fernandez de Rojas D, Virchow JC, et al. Respiratory allergy caused by house dust mites: What do we really know? J Allergy Clin Immunol. 2015; 136: 38-48.

18. Mehta AA, Shailaja M. Role of cytokines in pathophysiology of asthma. Iran J Pharm Ther. 2006; 5: 1-14.

19. Patel S, Meher BR. A review on emerging frontiers of house dust mite and cockroach allergy research. Allergol Immunopathol. 2016; 44: 580-93.

20. Molaae N, Mosayebi G, Pishdadian A, Ejtehadifar M, Ganji A. Evaluating the proliferation of human peripheral blood mononuclear cells using MTT assay. IJBMSP. 2017; 2: 25-8.

21. Buyuktiryaki B, Sahiner UM, Girgin G, Birben E, Soyer OU, Cavkaytar O, et al. Low indoleamine 2,3-dioxygenase activity in persistent food allergy in children. Allergy. 2015; 71: 258-66.

22. Munn DH, Mellor AL, Regents G, Place F. Regulation and tolerance. Allergy Asthma Immunol. 2017; 37: 193-207. 